cisplatin-resistant phenotype of A2780 cell line (BCARE A2780 3.3\% versus 39\% in A2780-cisplatin-resistant cells). The assay currently tests ex vivo patient derived cultures using a retrospective cohort of ovarian cancer patients. Correlations between BCARE scores and patient response to treatment will be investigated.

Conclusions Our BCARE-Score is capable of identifying dynamic alterations in HR-pathways as indicated by the differences observed in A2780 and cisplatin-resistant subline, which is not assessed by the current clinically applied HR assays. BCARE shows clear potential to be an effective tool in the prediction of primary drug response and more importantly in the detection of developed drug resistance.

\section{EPV198/\#424 LAPAROSCOPIC RESTAGING SURGERY FOR GYNAECOLOGICAL MALIGNANCIES}

${ }^{1} \mathrm{O}$ Puga*, 'M Urzua, ${ }^{2}$ E Pertossi. ${ }^{1}$ Hospital Dr Sotero del Rio, Obstetric and Gynecology, Santiago, Chile; ${ }^{2}$ Pontificia Universidad Catolica de Chile, Obstetric and Gynecology, Santiago, Chile

\subsection{6/ijgc-2021-IGCS.269}

Objectives To evaluate the feasibility and safety of laparoscopically staging patients with previous incomplete staged gynaecological cancers

Methods Patients without presurgical evidence of metastatic disease were laparoscopically reassessed. The procedure involved para-aortic and pelvic lymph node dissection and omentectomy for ovarian, fallopian tube and endometrial carcinoma; exclusive pelvic lymph node dissection for cervical carcinoma, oophorectomy and omentectomy for borderline tumors. Medical records were reviewed.

Results We performed 51 laparoscopic restaging surgeries: 14 ovarian cancer, 15 endometrial cancer, 17 borderline ovarian tumors, 4 cervical cancers and one fallopian tube carcinoma. Mean age was 48 years (16-70). In 39 patients the first surgery was performed by laparotomy. The mean body mass index was $28(20-40)$. Operative room time was $203 \mathrm{~min}(70-390)$ and mean postoperative hospital stay was 2 days. We performed 32 pelvic lymphadenectomies (average 15 lymph nodes), 30 para-aortic lymphadenectomies (8 lymph nodes), 27 omentectomies and 17 hysterectomies. Average estimated blood lost was $85 \mathrm{cc}$. There was one laparo-conversion for adhesions, one bowel injury, one cardiorespiratory arrest at recovery room and 2 lymphatics cystics. Lymph nodes and omentum were negative for metastasis. There were no patients up staged, in 9 endometrial and 9 ovarian cancers the complete negative restaging allowed us to decide that adjuvant therapy was not necessary. Five patients received adjuvant radiotherapy and 5 chemotherapy

Conclusions Laparoscopy is a feasibility technical option to perform restaging of gynaecological malignancies. Decreasing hospital stay, postoperative pain, few blood lost and low morbidity. Laparotomy for adhesions and risk of visceral injury may be anticipated.

\section{EPV199/\#430 CLINICAL AND SURVIVAL OUTCOMES OF MALIGNANT NON-DYSGERMINOMATOUS GERM CELL TUMOR OF OVARY: A SINGLE INSTITUTIONAL EXPERIENCE OF 64 PATIENTS}

${ }^{1} \mathrm{H}$ Mansouri, ${ }^{2} \mathrm{H}$ Salhi, ${ }^{3,4}$ I Zemni ${ }^{*},{ }^{1} \mathrm{~L}$ Achouri, ${ }^{3,4} \mathrm{MA}$ Ayadi, ${ }^{3} \mathrm{~K}$ Rahal. ${ }^{1}$ Regional Hospital of Jendouba, University of Tunis ELManar Tunisia, Department of Surgical Oncology, Jendouba, Tunisia; ${ }^{2}$ Regional Hospital of Siliana, University of Tunis ELManar Tunisia, Department of Surgical Oncology, Siliana, Tunisia; ${ }^{3}$ Salah Azaiez Institute of Cancerology, University of Tunis El Manar, Department of Surgical Oncology, Tunis, Tunisia; ${ }^{4}$ University of Tunis El Manar, Sciences Faculty of Tunis, Laboratory of Microorganismes and Active Biomolecules, Tunis, Tunisia

\subsection{6/ijgC-2021-IGCS.270}

Objectives To evaluate clinicopathological features and survival outcomes of malignant non-dysgerminomatous germ cell tumor (MNDGCT) of the ovary.

Methods We retrospectively recorded clinicopathological and therapeutic data of 64 patients with MNDGCT of ovary treated at the Salah Azaiez Institute of Tunisia between 1970 and 2012.

Results The median age was 26 years (range7-75 years). The most frequent subtype was immature teratoma $(n=27$, $42.18 \%)$ followed by yolk-sac tumor $(n=15,23.43 \%)$ and mixed germ cell tumor $(n=11,17.18 \%)$. Most of the patients had stage I and II disease (41 cases, 64.1\%) while 17 (26.6\%) and $6(9.3 \%)$ were staged III and IV disease, respectively. Radical surgery was performed in 23 patients $(35.8 \%)$ and conservative surgery in 41 patients(64.2\%) associated with lymph node dissection in 19 cases. Complete macroscopic resection was obtained in 48 patients (78.68\%) and lymph node metastasis was observed in $41.5 \%$ of cases. Adjuvant chemotherapy was indicated in cases in $54.68 \%$ of cases. After a mean time follow-up of 74 months (7-182 months), complete remission was observed in 47 patients. The 5 -year progression-free survival (PFS) was $73.5 \%$.The 5 year overall survival (OS) was $82.23 \%$ and was significantly decreased in young patients $\leq 15$ years $(49.5 \%$ vs $89.4 \% ; \mathrm{p}=0.003)$, advanced stage $(94.6 \%$ in stage I-II vs $59.8 \%$ in stage III-IV; $\mathrm{p}=0.01)$ and macroscopic residual disease $(88.9 \%$ vs $52.9 \%$; $\mathrm{p}=0.02)$.No difference in OS was noted following stratification by tumor size $\leq$ or $>20 \mathrm{~cm}(84.7 \%$ vs $74.6 ; \mathrm{p}=0.44)$ and conservative or radical surgery $(89.8 \%$ vs $70 \% ; \mathrm{p}=0.34)$

Conclusions Macroscopic residual disease as well as advanced FIGO stage and age are the main prognostic factors in MNDGCT.

\section{EPV200/\#432 CLINICAL AND SURVIVAL OUTCOMES OF PURE DYSGERMINOMA OF OVARY: A SINGLE INSTITUTIONAL EXPERIENCE OF 31 PATIENTS}

${ }^{1} \mathrm{H}$ Salhi, ${ }^{2} \mathrm{H}$ Mansouri, ${ }^{3,4} \mathrm{MA}$ Ayadi, ${ }^{2} \mathrm{~L}$ Achouri, ${ }^{3,4} \mathrm{I}$ Zemni*, ${ }^{3} \mathrm{~K}$ Rahal. ${ }^{1}$ Regional Hospital of Siliana, University of tunis ELManar Tunisia, Department of Surgical Oncology, Siliana, Tunisia; ${ }^{2}$ Regional Hospital of Jendouba, University of tunis ELManar Tunisia, Department of Surgical Oncology, Jendouba, Tunisia; ${ }^{3}$ Salah Azaiez Institute of Cancerology, University of Tunis El Manar, Department of Surgical Oncology, Tunis, Tunisia; ${ }^{4}$ Sciences Faculty of Tunis, University of Tunis El Manar, Laboratory of Microorganismes and Active Biomolecules, Tunis, Tunisia

10.1136/ijgc-2021-IGCS.271 
Objectives To evaluate clinicopathological features and survival outcomes of pure dysgerminoma of the ovary

Methods We retrospectively recorded clinicopathological and therapeutic data of 31 patients with pure dysgerminoma of ovary treated at the Salah Azaiez Institute of Tunisia between 1970 and 2012.

Results The median age was 22 years (12-60 years). The distribution of patients according FIGO stage was as follow:stage I:16 (51.6\%),stage II:3 (9.7\%),stage III: 11(35.5\%) and stage IV:1 case.Radical surgery was performed in 11 patients and conservative surgery in 20 patients (64.5\%) associated with node picking lymphadenectomy in 7 cases and complete lymphadenectomy in 7 cases. Complete macroscopic resection was obtained in 22 cases (70.96\%) and lymph node metastasis was observed in $51.1 \%$ of cases. Adjuvant chemotherapy was indicated in 15 cases and adjuvant radiotherapy in 10 cases. After a mean time follow-up of 74 months (7-182 months), complete remission was observed in 26 patients. The 5 -year progression-free survival (PFS) was $85.2 \%$. The 5 -year overall survival (OS) was $89.5 \%$ and was significantly decreased in the advanced stage $(100 \%$ in stage I-II vs $75 \%$ in stage III-IV; $\mathrm{p}=0.02)$. There was a significant difference in OS and PFS between complete resection and residual disease groups (100\% vs $67.5 \% ; \mathrm{p}=0.03$ and 88.9 vs $75 \% ; \mathrm{p}=0.03$, respectively). No difference in OS and PFS was noted following stratification by age $\leq$ or $>15$ years $(\mathrm{p}=0.36$ and $\mathrm{p}=0.1)$, tumor size $\leq$ or $>20 \mathrm{~cm}(\mathrm{p}=0.27$ and $\mathrm{p}=0.68)$ and conservative or radical surgery $(\mathrm{p}=0.87$ and $\mathrm{p}=0.17)$.

Conclusions Macroscopic residual disease, as well as advanced FIGO stage, were the main prognostic factors in pure dysgerminoma of the ovary.

\section{EPV201/\#44 REAL-WORLD DATA ANALYSIS OF SECOND-LINE POLY (ADP-RIBOSE) POLYMERASE INHIBITOR MAINTENANCE THERAPY IN PATIENTS WITH ADVANCED OVARIAN CANCER}

${ }^{1} \mathrm{R}$ Reid*, ${ }^{2} \mathrm{Y} \mathrm{Xie}_{\mathrm{e}}{ }^{2} \mathrm{~J}$ Shi, ${ }^{3} \mathrm{~K}$ Wallace. ' US Oncology, Virginia Cancer Specialists, Medical Oncology, Fairfax, USA; ${ }^{2}$ Ontada, Real World Research, The Woodlands, USA; ${ }^{3}$ Clovis Oncology, Inc., Health Economics and Outcomes Research, Boulder, USA

\subsection{6/ijgC-2021-IGCS.272}

Objectives Poly(ADP-ribose) polymerase inhibitors (PARPis) have been recommended since 2017 as second-line (2L) maintenance treatment by the NCCN for ovarian cancer (OC) patients with or without BRCA1 or BRCA2 (BRCA) alterations. Here, we assessed PARPi use from real-world data.

Methods From the iKnowMed electronic health record database of the US Oncology Network (>470 sites), adult females were included if they were diagnosed with advanced OC, received a $2 \mathrm{~L}$ platinum-containing regimen for advanced OC, and had $\geq 2$ visits between 1 January 2016 and 1 July 2020 . Patients were followed until 31 October 2020, last patient record, or death, whichever occurred earliest. A 24-month landmark survival analysis was performed.

Results Out of 11,494 patients diagnosed with advanced OC, 1051 met the inclusion criteria; 513/1051 (49\%) subsequently received any maintenance therapy (table 1 ). The proportion of patients receiving 2L PARPi maintenance increased from 17\% in 2018 to $34 \%$ in 2019 but decreased to $22 \%$ in 2020 (figure 1). Among $\mathrm{BRCA}^{+}$patients, 33\% (46/140) received 2L PARPi maintenance, while documented $\mathrm{BRCA}^{-}$patients received PARPi maintenance at a significantly lower rate $(23 \%$; $155 /$
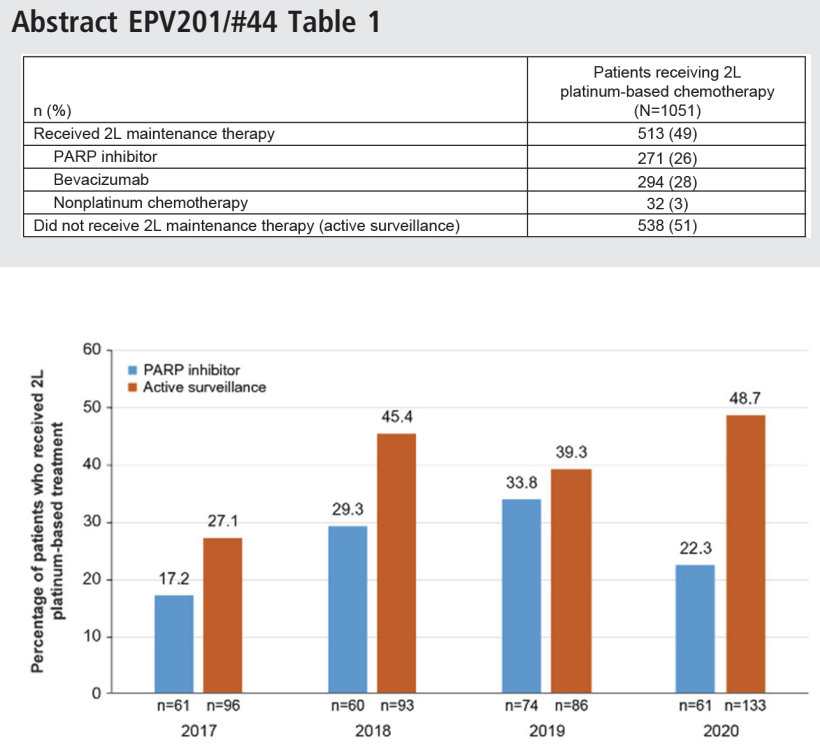

Abstract EPV201/\#44 Figure 1 Propotion of patients receiving 2L PARPi maintenance (active surveillance)

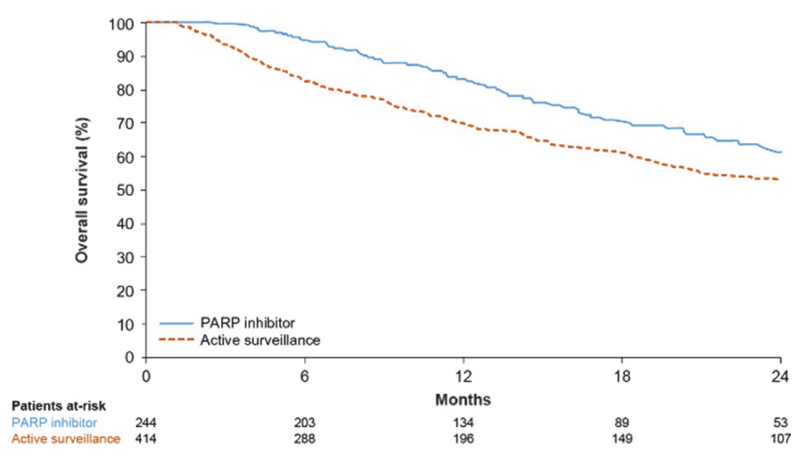

Abstract EPV201/\#44 Figure 2 Overall survival

622; $\mathrm{P}=0.0192$ ). Survival at 24 months was significantly higher with PARPi maintenance vs active surveillance: $61.2 \%$ (95\% CI, 52.4\%-68.8\%) vs 53.0\% (95\% CI, 47.1\%-58.7\%; log-rank $\mathrm{P}=0.0045$ ) (figure 2).

Conclusions Our data suggest a significant proportion of eligible patients are not receiving $2 \mathrm{~L}$ maintenance therapy despite treatment guideline recommendations and apparent survival benefits associated with its use.

\section{EPV202/\#446 SENTINEL LYMPH NODE IDENTIFICATION IN EARLY STAGE OVARIAN CANCER: IS IT STILL POSSIBLE AFTER PRIOR TUMOR RESECTION?}

${ }^{1} \mathrm{P}$ Laven*, ${ }^{2} \mathrm{R}$ Kruitwagen, ${ }^{2} \mathrm{~S}$ Lambrechts, ${ }^{3} \mathrm{~T}$ Van Gorp, ${ }^{4} \mathrm{~B}$ Slangen, ${ }^{5} \mathrm{P}$ Zusterzeel, ${ }^{6} \mathrm{~J}$ Van Der Pol. 'Maastricht Universitair Medisch Centrum, Gynaecology, Maastricht, Netherlands; ${ }^{2}$ Maastricht University Medical Centre, Gynecology, Maastricht, Netherlands; ${ }^{3}$ University Hospital Leuven, Gynaecological Oncology, Leuven, Belgium; ${ }^{4}$ Maastricht University Medical Center, Gynaecology and Obstetrics, Maastricht, Netherlands; ${ }^{5}$ Radboud Univeristy Hospital, Gynaecological Oncology, Nijmegen, Netherlands; ${ }^{6}$ Maastricht Universitair Medisch Centrum, Nuclear Medicine, Maastricht, Netherlands

\subsection{6/ijgc-2021-IGCS.273}

Objectives Sentinel lymph node (SLN) detection in ovarian cancer is feasible when tracers are injected before the pathological ovary is resected. This study aims to investigate 